\title{
Erratum: Noncyclic Berry phase and scalar Aharonov-Bohm phase for the spin-redirection evolution in an atom interferometer [Phys. Rev. A 86, 022105 (2012)]
}

\author{
Atsuo Morinaga and Kota Nanri \\ (Received 4 July 2016; published 29 July 2016)
}

DOI: 10.1103/PhysRevA.94.019907

The following three points in the paper should be corrected.

(1) In the paper, we discussed the noncyclic Berry phase as a function of the cone angle $\theta$ between the rotation axis $(+z$ axis) and the direction of the magnetic field in the range of $0 \leqslant \theta \leqslant \pi$. In the previous Erratum [1], we presented a formula of a noncyclic Berry phase for $\pi / 2 \leqslant \theta \leqslant \pi$. However, the formula was ambiguous and should be corrected. Here we present a consistent formula for the noncyclic Berry phase. In our recent papers [2,3], we stated that the Berry phase at an angle $\theta$ larger than $\pi / 2$ is identical to that with a reversed rotation direction at angle $\pi-\theta$ because its rotation axis should be reversed to the $-z$ axis. According to the definition presented by Welte $e t$ al. [4], we define that the sign of $\theta$ is positive for the right-handed rotation of the magnetic field around the positive $z$ axis, whereas it is negative for the left-handed rotation. On the other hand, the rotation angle $\phi$ of the magnetic field is defined by the magnitude $(0 \leqslant \phi \leqslant 2 \pi)$, despite the sense of the rotation. Then, the signed solid angle $\Omega$ for a rotation angle $\phi$ is described by the following consistent equation for $-\pi / 2 \leqslant \theta \leqslant \pi / 2$ :

$$
\begin{aligned}
\Omega(\theta, \phi)= & \operatorname{sgn}(\theta) \\
& \times \begin{cases}2 \sin ^{-1}\left(\frac{\sin (\phi / 2)}{\sqrt{1+\tan ^{2} \theta \cos ^{2}(\phi / 2)}}\right)-\phi \cos \theta, & 0 \leqslant \phi \leqslant \pi, \\
2\left[\pi-\sin ^{-1}\left(\frac{\sin (\phi / 2)}{\sqrt{1+\tan ^{2} \theta \cos ^{2}(\phi / 2)}}\right)\right]-\phi \cos \theta, & \pi \leqslant \phi \leqslant 2 \pi .\end{cases}
\end{aligned}
$$

The signed solid angle is shown in Fig. 1 of this Erratum for various $\theta$ 's. Then, the noncyclic Berry phase between the lower $m$ state and the upper $m^{\prime}$ states is given by

$$
\gamma(\theta, \phi)=-\left(m^{\prime}-m\right) \Omega(\theta, \phi),
$$

which is independent of the magnitude and sign of the $g$ factors [2-5]. Consequently, Eq. (4) in the paper should be corrected for $-\pi / 2 \leqslant \theta \leqslant \pi / 2$ as follows:

$$
\begin{aligned}
\gamma(\theta, \phi)= & -\operatorname{sgn}(\theta)\left(m^{\prime}-m\right) \\
& \times \begin{cases}2 \sin ^{-1}\left(\frac{\sin (\phi / 2)}{\sqrt{1+\tan ^{2} \theta \cos ^{2}(\phi / 2)}}\right)-\phi \cos \theta, & 0 \leqslant \phi \leqslant \pi, \\
2\left[\pi-\sin ^{-1}\left(\frac{\sin (\phi / 2)}{\sqrt{1+\tan ^{2} \theta \cos ^{2}(\phi / 2)}}\right)\right]-\phi \cos \theta, & \pi \leqslant \phi \leqslant 2 \pi .\end{cases}
\end{aligned}
$$

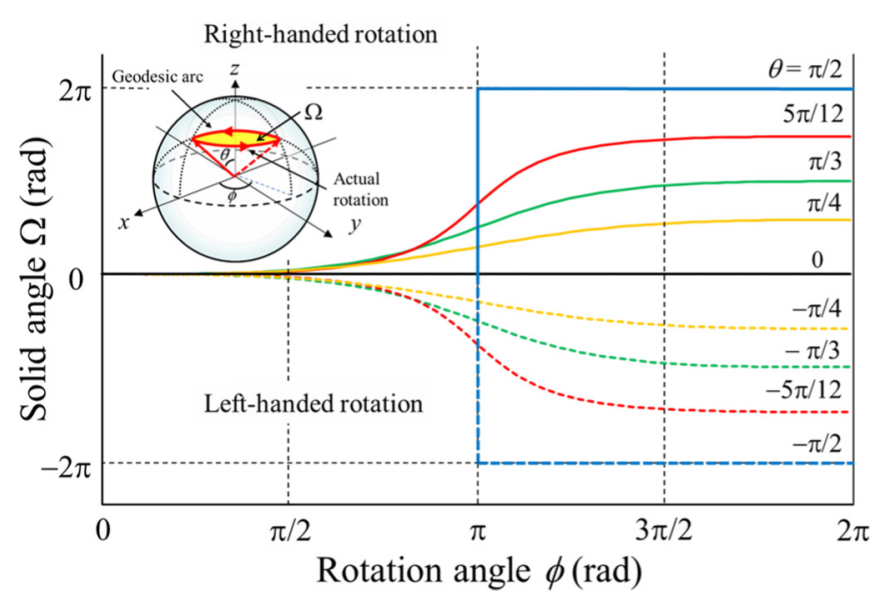

FIG. 1. Solid angle for the partial rotation of the magnetic field. $\phi$ : rotation angle. $\theta$ : cone angle between the rotation axis $(+z$ axis) and the magnetic field. 
Certainly, the experimental results of the noncyclic Berry phase for $m=-1$ and $m^{\prime}=1$ are in good agreement with Eq. (3) of this Erratum as shown in Fig. 7 of the paper if the value of $\theta$ larger than $\pi / 2$ is replaced with $-(\pi-\theta)$. Furthermore, the noncyclic Berry phase at $\phi=2 \pi$ is consistent with the Berry phase for a whole rotation, namely,

$$
\gamma(\theta)=-\operatorname{sgn}(\theta)\left(m^{\prime}-m\right) 2 \pi(1-\cos \theta), \quad-\pi / 2 \leqslant \theta \leqslant \pi / 2 .
$$

Thus, Fig. 7 in the previous Erratum [1] should be withdrawn.

(2) In Sec. IV A of the paper, we stated that the sinusoidal shift observed in the frequency shift arises from the Doppler shift. However, this statement was wrong. The sinusoidal variation occurs from the overlapping of spectra of the carrier and sideband components generating within the Fourier width of the observation period under the rotation of the magnetic field [2].

(3) At the end of the third paragraph in Sec. IV B of the paper, we stated that "Thus, the solid angle is ... a polar gauge as discussed in Ref. [12]." These sentences should be corrected to "Thus, the solid angle is the area of the spherical rectangle enclosed by the arc $A B$, the great circle from $B$ to the equator, a part of the equator, and the other great circle from the equator to $A$. Namely, it means the phase was measured using a rotation gauge as discussed in Ref. [12]."

[1] A. Morinaga and K. Nanri, Phys. Rev. A 88, 039904(E) (2013).

[2] A. Morinaga and Y. Hasegawa, Phys. Rev. A 92, 013403 (2015).
[3] A. Morinaga and Y. Hasegawa, Phys. Rev. A 93, 059902(E) (2016).

[4] S. Welte, C. Basler, and H. Helm, Phys. Rev. A 89, 023412 (2014).

[5] M. V. Berry, Proc. R. Soc. London, Ser. A 392, 45 (1984). 